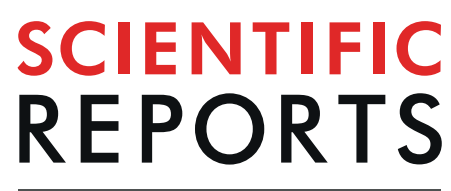

natureresearch

Check for updates

\title{
On the use of a continuous thrust to find bounded planar trajectories at given altitudes in Low Earth Orbits
}

\author{
Allan Kardec de Almeida Jr. $\mathbb{1}^{1,2} \llbracket$, Jhonathan Orlando Murcia Piñeros $\mathbb{D}^{1} \&$ \\ Antonio Fernando Bertachini de Almeida Prado $\mathbb{1}^{1}$
}

In this work, it is shown how a spacecraft equipped with a thrust and subjected to a drag force can be bounded at specific altitudes as function of the parameters of the thrust. It is used nonlinear dynamics tools to find attractors, which bound the motion of the spacecraft. For a specific set of parameters of the thrust, the spacecraft is bounded to a given altitude. Several forms for the thrusts are proposed in order to bound the altitude of the spacecraft. The influence of several forms of perturbations in the altitude of the spacecraft is also investigated in this work, like the solar radiation pressure, gravity of the Moon and oblateness of the Earth. Finally, nonlinear dynamics tools are also used to investigate transfers among the bounded orbits in different altitudes.

The number of satellites in Low Earth Orbits (LEOs) is increasing due to the development of Small Sats missions. Over 1,000 smallsats were launched from 2012 to 2017, according to [Smallsats by the Numbers 2018, Bryce Space and Technology]. Satellites in the Low Earth Orbits (LEOs) are highly perturbed by drag ${ }^{1}$, among other forces. The atmospheric drag can be used in maneuvers around the Earth and planets like Mars or Venus ${ }^{2-4}$. In general, some kind of thrust is necessary to counteract the losses of altitude and energy due to the atmospheric drag. Orbit maintenance may be performed by using continuous or impulsive maneuvers to return the satellite to near its initial orbit. In the present work, several forms of continuous thrusts are defined and studied with the objective to select the ones that give trajectories that are bounded ${ }^{5}$ at specific altitude regions. In that sense, the present paper proposes solutions to keep a spacecraft in a given orbit by applying a thrust that continuously compensate the perturbations received from the environment. Depending on the perturbation, the spacecraft is bounded to a circular orbit or to an elliptic, but periodic orbit in a proper reference frame. Regarding the applied control, several forms are studied. The most complete one has a component proportional to the square of the velocity of the spacecraft, to compensate the effect of drag; another component proportional to the altitude of the spacecraft, to be able to adjust the orbit to fit a desired altitude; and one more component proportional to the ratio of the velocity and the altitude of the spacecraft, to give more controllability to the spacecraft by adding one more degree of freedom to the system. A similar concept is applied in science missions, which goal is to measure the gravitational field, the so called drag-free missions, which are characterized by the use of control techniques to reduce the disturbances affecting the spacecraft, mainly drag. Some examples of these missions are available in references ${ }^{6-8}$.

Although dissipative forces like drag require consumption of fuel to be balanced, in terms of nonlinear dynamics, it enables the existence of attractors ${ }^{9,10}$. In this work, the drag is used to help the mission, because we take advantage of the limited boundaries of the attractors to keep the trajectories in desired specific altitude regions. The main objective of this work is to use attractors to bound trajectories in strategic regions of the atmosphere. In order to better study these attractors, a Poincaré section ${ }^{11}$ will be used to reduce the dimension of the system and to locate it in the phase-space domain.

Besides drag, other perturbative forces also influences the motion at LEOs, like the ones that come from the solar radiation pressure, the oblateness of the Earth and the gravitational attraction of the Moon. Thus, additionally, the influence of these perturbations over the attractors - their displacement in the phase-space - will also be investigated.

Finally, we introduce a method to interpret the successfulness of a transfer for a spacecraft among attractors with different altitudes by the use of their basins of attraction in the phase-space of the Poincaré section. The idea 
of this technique is to combine a transfer orbit with the requirements to keep the spacecraft in a given attractor. Changing the parameters of the control remove the spacecraft from its attractor and, under some conditions, send it to another attractor. The present study maps these conditions, showing the destiny of the spacecraft after a change in those parameters. It may go to another attractor, to reentry the atmosphere of the Earth or to leave the orbit of the Earth.

The system used in this work is based in the Tsien problem ${ }^{12}$, which represents a satellite orbiting the Earth subjected to a thrust. A dissipative force will be introduced in the system, which represents the drag force that a satellite is subjected due to the atmosphere density. This dissipative force will be used to send the system to specific attractors in desired altitudes of the atmosphere. In a consecutive analysis, perturbative forces will be included, which represent the effects due to the solar radiation pressure over the satellite, the $J_{2}$ potential term due to the oblateness of the Earth and the perturbation due to the gravitational interaction with the Moon. The effects of these additional perturbations over the attractors are investigated through their dependence on the values of the parameters of the perturbed system.

The problem of stability of the attractors is also considered. A study is made to show the linear stability of the attractors, helping to decide if it is a good location to place a spacecraft.

Note that in the present research, the orbital maintenance is not applied in the traditional way, i.e., to return the satellite to the operational orbit after some time to compensate the effects of perturbations such that the fuel consumption is minimized, with multiple impulses periodically or low thrust applied in the most efficient regions. The problem under study is how to apply a continuous thrust to keep the spacecraft in a given altitude, not allowing oscillations in the orbit that may minimize fuel consumption. Such situation may be required in a mission, or in part of mission, where observations are required to be made from a given fixed range of altitude. In this case, it is applied a continuum force to transport the satellite into an attractor that lies in the desired altitude, maintaining it in this region under the continuum influence of several types of perturbations. Then, the continuous thrust is necessary to keep the satellite in this region. To do that, one of the best technological methods available is the application of low thrust with electrical propulsion systems. Of course it is possible to find other strategies that may consume less fuel, but they do not keep the spacecraft in a fixed altitude, which may be required sometimes.

In Sec. 2, the models used to formulate the problem are shown. The results for the satellite subjected to drag, perturbations and different forms of thrusts are showed in Sec. 3.

\section{Formulation of the problem}

The Tsien problem ${ }^{12}$ consists in a set of two bodies: a main central body and a second one with negligible mass (the spacecraft) moving around the center of mass of the central body and subjected to a thrust. Additionally to the thrust, a dissipative drag force $(\vec{D})$ due to the atmospheric interaction with the spacecraft is always considered in the problem. Other perturbations are also taken into consideration for some cases. Using an inertial frame of reference centered in the Earth, the equation of motion of such a spacecraft is

$$
\vec{r}=-\mu \frac{\vec{r}}{r^{3}}-\beta v \vec{v}+\tau \vec{\theta}+\vec{P},
$$

where $\vec{r}$ is the position vector of the spacecraft with magnitude $r$. The velocity vector is defined by $\vec{v}=d \vec{r} / d t$, with magnitude $v$. $\tau$ is the magnitude of the specific thrust to be studied; $\vec{\theta}$ is a unit vector perpendicular to $\vec{r}$, defined according to the traditional polar coordinates; $\mu$ is the gravitational parameter of the main body and $\beta v \vec{v}$ is the term due to the drag, which is in the opposite direction to the velocity $\vec{v}$ of the spacecraft; $\beta=\rho B$, where $\rho$ is the atmosphere density and $B$ is the Ballistic Coefficient $\left(B=A C_{D} / 2 \mathrm{~m}\right)$, where $C_{D}$ is the drag coefficient, $A$ the transversal area and $m$ is its mass ${ }^{13}$. In this work, $\rho$ is assumed to be constant for low oscillations of the altitude, around $600 \mathrm{~km}$. The density is evaluated using the model NRLMSISE- $00^{14}$ for $600 \mathrm{~km}$. The vector $\vec{P}$ represents the perturbations due to Solar Radiation Pressure, oblateness of the central body (Earth) or third body influence (Moon). These perturbations are better described next.

The Oblateness of the Earth modelled with the term of the acceleration due to $J_{2}$ - the second term of the gravity potential of the Earth ${ }^{1}$ - is considered in this work. In this way, the gravitational potential of the Earth is given by

$$
U=-\frac{\mu}{r}-\frac{3 J_{2} \mu}{2 r} \frac{r_{e}^{2}}{r^{2}}\left(\sin ^{2} \phi-\frac{1}{3}\right),
$$

where $\phi$ is the latitude and $r_{e}$ the mean equatorial radius. The planar orbits analyzed here are restricted to the equatorial and polar planes ( $\phi=0$ and $\phi= \pm \pi / 2$, respectively), which are two of the most important cases, among all the values of $\phi$.

The acceleration due to a thrust given by a planar solar sail attached to the satellite coming from the solar radiation pressure is given by ${ }^{15}$

$$
\vec{P}_{S R P}=\delta \frac{A}{m} \frac{k \cos ^{2}(\gamma)}{\|\vec{r}\|^{2}} \vec{n},
$$

where $\delta$ is a positive non-dimensional parameter less than 1 ( $\delta=1$ means a perfect reflection); $k$ is a parameter that depends on the luminosity of the body $\left(k=2 p_{e} R^{2}\right.$ in the case where the Sun is the source, where $p_{e}=4.56 \times 10^{-6} \mathrm{~kg} /\left(\mathrm{ms}^{2}\right)$ holds for the solar radiation pressure at a Sun-Earth distance from the Sun $) ; A$ is the area of the flat solar sail; $m$ is the mass of the satellite; $\vec{r}_{s}$ is the vector that locates the sail from the source of photons; 
$\vec{n}$ is the vector normal to the solar sail and $\gamma$ is the angle between $\overrightarrow{r_{s}}$ and $\vec{n}$, which is given according to $\cos \gamma=\left(\vec{r} \cdot \vec{n} / r_{s}\right)$. In our evaluations, $\vec{n}$ is set in the direction of $r_{s}$, the ratio area-to-mass is $5.562 \mathrm{~m}^{2} / \mathrm{kg}$ and $\delta=0.0026$. The parameter $\delta$ is used to represent many forms of practical applications, such as a satellite which reflexivity of its panel can be changed ${ }^{16,17}$, as well as a satellite which effective area is adjustable ${ }^{18,19}$. The source of photons (the Sun) is assumed to rotate around the Earth in a circular orbit. However, the vector $\overrightarrow{r_{s}}$ is a function of time, so the equations of motion are explicit functions of time, which means that the dimension of the phase-space will be increased by one. In order to avoid this issue, a rotating frame of reference that rotates around the Earth with the same angular velocity of the Sun is used. This system does not depend explicitly on time anymore and the dimension of the phase-space is unchanged taking into account the perturbation. Additionally, the solar rays are assumed to be parallel in the vicinity of the Earth, thus a shadow function is included, which means that the solar pressure effects are turned off while the spacecraft passes behind the Earth with respect to the Sun.

The gravity field of the Moon is also included in the system. Our model assumes that the Moon is rotating around the Earth in a circular orbit. Once again, the gravitational term due to the Moon would be explicitly time dependent, thus the system is written in a rotating frame of reference that rotates with the same angular velocity of the Moon. In this new frame of reference, the equations of motion are not explicitly dependent on time, so the dimension of the phase-space is unchanged in comparison with the unperturbed case, when the perturbation of the Moon is not applied. The satellite is assumed to be in an orbit co-planar to the Earth-Moon orbital plane.

These perturbations represent the highest forces at $\mathrm{LEO}^{1,20}$ and they are taken into consideration individually. The equation of motion is numerically integrated through a Runge Kutta F-7/8 21 numerical integrator. A Poincaré section is analyzed to get a better visualization of the attractors in the phase-space. The Poincaré section is defined in the right half-plane $y=0$. In the case studied here, the motion is restricted to a plane, thus the Poincaré section is defined where the trajectory crosses the value $y=0$ in the positive side of the $x$ axis, which value is $y=0$. It means that the phase-space in this section has three dimensions. Unless stated otherwise, some other considerations are used in this paper, as show next.

- The spacecraft is initially inserted in a Keplerian circular orbit of radius $a_{0}$ around the Earth, so its velocity is given by $v_{0}=\sqrt{\mu / r_{0}}$.

- The altitude alt is defined as $r-r_{e}$, where $r_{e}=6378.136 \mathrm{~km}$ is the mean equatorial radius of the Earth.

- To show the parameters in a more suitable form in the results, a unit of length $(u L)$ is defined as $u L=6978.136 \mathrm{~km}$ and the unit of time is such that the period of a keplerian orbit with semi-major axis $u L$ is $2 \pi$, so $u T=\sqrt{(u L)^{3} / \mu}$.

- In those equations, $\mu$ is the gravitational parameter of the Earth. To have an idea of the parameter $\beta$, for the International Space Station it is $\beta_{\text {ISS }}=1.53055116 \times 10^{-14} \mathrm{~m}^{-1}$ for altitudes around $600 \mathrm{~km}$, which corresponds to a ratio area-to-mass $0.005562 \mathrm{~m}^{2} / \mathrm{kg}$. To keep the transient in a reasonable time scale, in the order of a few years, the parameter $\beta$ is fixed in $\beta=\beta_{I s s} \times 10^{3}$, which corresponds to a spacecraft with the same drag coefficient $C_{D}$ of the ISS, but an area-to-mass ratio $5.562 \mathrm{~m}^{2} / \mathrm{kg}$. The ISS is used as a reference, because it is the largest artificial satellite in LEO. Due to its area-to-mass ratio, it has a rapid decay and, to maintain the ISS in orbit, impulsive maneuvers are made periodically.

Note that basics properties of nonlinear dynamics tools are used in this paper to search for orbits at LEOs, like Poincaré sections, attractors and their basin of attraction for the dynamical system considered. Attempts to rigorous definition of such tools are extensively available in the literature ${ }^{9-11}$.

\section{Results}

For all kinds of thrusts, the case where there are no perturbations acting in the system is initially investigated, i.e. $\vec{P}=\overrightarrow{0}$ in Eq. 1, where $\overrightarrow{0}$ is the null vector. The system will be then subjected to several types of perturbations: the solar radiation pressure ${ }^{15}$, the $J_{2}$ term of the gravitational potential of the Earth ${ }^{1}$ for polar and equatorial orbits and the gravitational interaction with the Moon ${ }^{20}$. This process will be done for several forms of thrust. Firstly, two forms for the thrust will be independently investigated in subsections 3.1 and 3.2. Then, a new form that includes all the terms added will be proposed and investigated in subsection 3.3.

Thrust proportional to the radius. The first form of the thrust to be investigated is the case where the magnitude of the thrust is proportional to the radius. In this case, $\tau$ is given by

$$
\tau=\alpha_{1}\left(r_{0}-r\right)+\alpha_{0} \beta v_{0}^{2},
$$

where $\alpha_{1}$ and $\alpha_{0}$ are parameters of the magnitude of the thrust and $r_{0}$ is a parameter that should be chosen around the desired altitude of the attractor. The second term is a simple constant and its form is selected to approximately compensate the magnitude of the drag in the case where the parameter $\alpha_{0}$ equals a unity, since the magnitude of the initial velocity $v_{0}$ is near the magnitude of the velocity of the spacecraft in the attractor. The first term of the right side of Eq. (4) is a linear proportional control where $\alpha_{1}$ is the proportional gain ${ }^{22}$. Note that it can be seen as a spring-mass system, having a force that obligates the spacecraft to oscillate around $r_{0}$. In the case where the spacecraft is falling to the Earth, i.e. $r<r_{0}$, the force is approximately in the same direction of the velocity, hence its magnitude tends to increase and the spacecraft tends to ascend. On the other side, in the case where the spacecraft is escaping from the Earth, i.e. $r>r_{0}$, the force is approximately in the opposite direction of the velocity, hence the spacecraft tends to descend. Thus, due to the existence of the drag, the system shall work like a damped oscillator. 


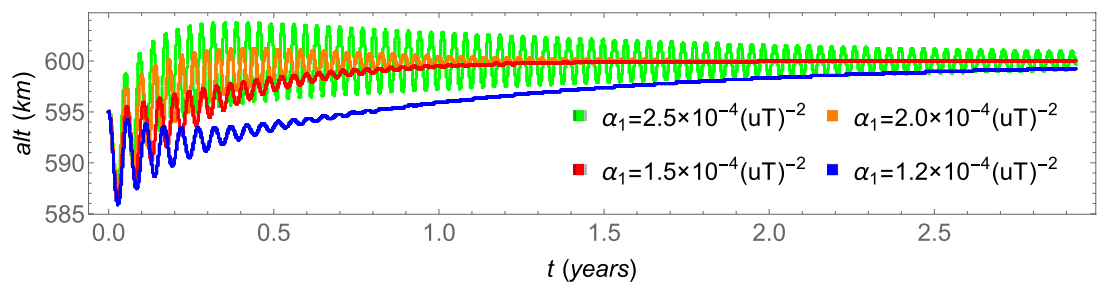

Figure 1. The evolutions to the same attractor for different values of the parameter $\alpha_{1}$. The overdumped pattern cases are shown in blue and red, while the underdumped pattern cases are shown in orange and green. The other parameters are $\alpha_{0}=1, r_{0}=r_{e}+600 \mathrm{~km}$ and $a_{0}=r_{0}-5 \mathrm{~km}$.

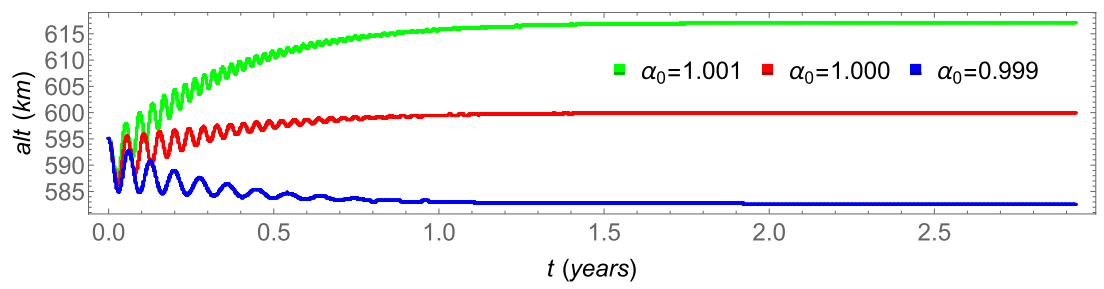

Figure 2. The final altitudes for different values of the parameter $\alpha_{0}$. The other parameters are $\alpha_{1}=1.5 \times 10^{-4}\left(u T^{-2}\right), r_{0}=r_{e}+600 \mathrm{~km}$ and $a_{0}=r_{0}-5 \mathrm{~km}$.

\begin{tabular}{|l|l|l|l|l|}
\hline \multirow{5}{*}{} & \multicolumn{5}{|l|}{ values for the parameter $\boldsymbol{\alpha}_{\mathbf{1}}\left(\mathbf{1 0}^{-4}(\boldsymbol{u} T)^{-2}\right)$} \\
\cline { 2 - 5 } & $\alpha_{\mathbf{1}}=\mathbf{1 . 2}$ & $\alpha_{\mathbf{1}}=\mathbf{1 . 5}$ & $\boldsymbol{\alpha}_{\mathbf{1}}=\mathbf{2 . 0}$ & $\boldsymbol{\alpha}_{\mathbf{1}}=\mathbf{2 . 5}$ \\
\hline$x\left(10^{6} \mathrm{~m}\right)$ & 6.978135999988396 & 6.978135999998476 & 6.978135999995473 & 6.978135999996812 \\
\hline$y(m)$ & $|y|<10^{-9}$ & $|y|<10^{-9}$ & $|y|<10^{-9}$ & $|y|<10^{-9}$ \\
\hline$\dot{x}(\mathrm{~m} / \mathrm{s})$ & $|\dot{x}|<10^{-8}$ & $|\dot{x}|<10^{-8}$ & $|\dot{x}|<10^{-8}$ & $|\dot{x}|<10^{-8}$ \\
\hline$\dot{y}(\mathrm{~m} / \mathrm{s})$ & 7557.86574523 & 7557.86574523 & 7557.86574523 & 7557.86574523 \\
\hline
\end{tabular}

Table 1. Coordinates of the attractors in the phase-space of the Poincaré section.

The parameters $\alpha_{0}$ and $r_{0}$ are set to $\alpha_{0}=1$ and $r_{0}=r_{e}+600 \mathrm{~km}$ (or $r_{0}=1 \mathrm{uL}$ ), which corresponds to an altitude equals to $600 \mathrm{~km}$. The altitudes as functions of time are shown in Fig. 1, for different values of the parameter $\alpha_{1}$.

The spacecraft evolves to the attractors, which coordinates in the phase-space of the Poincaré section are shown in Table 1 for several values of the parameter $\alpha_{1}$. Note that the coordinates of all the attractors are almost coincident, but the spacecraft evolves to this attractor in different ways, as shown in Fig. 1. The patterns are analogous to the solutions of the dumped harmonic oscillator: the overdumped pattern cases are shown in blue and red for the parameters $\alpha_{1}=1.2 \times 10^{-4}\left(u T^{-2}\right)$ and $\alpha_{1}=1.5 \times 10^{-4}\left(u T^{-2}\right)$, respectively, and the underdumped patterns cases are shown in orange and green, for the parameters $\alpha_{1}=2.0 \times 10^{-4}\left(u T^{-2}\right)$ and $\alpha_{1}=2.5 \times 10^{-4}\left(u T^{-2}\right)$, respectively. The time to reach the attractor is lower for values of the parameter closer to $\alpha_{1} \approx 1.8 \times 10^{-4}\left(u T^{-2}\right)$. For values of the parameter $\alpha_{1}<1.1 \times 10^{-4}\left(u T^{-2}\right)$, the first term of the right side of Eq. 4 is not strong enough to send the spacecraft to the attractor and, as a consequence, the spacecraft falls to the Earth. On the other side, for values of the parameter $\alpha_{1}>2.7 \times 10^{-4}\left(u T^{-2}\right)$, the first term of the right side of Eq. 4 is too strong in comparison with the drag, so that the amplitudes of the oscillations become larger along time and, as a consequence, the spacecraft cannot reach the attractor as well. On the other hand, the effect of the parameter $\alpha_{0}$ over the altitude of the attractors is shown in Fig. 2, which shows the altitude of the spacecraft for several values of $\alpha_{0}$ in the case where $\alpha_{1}=1.5 \times 10^{-4}\left(u T^{-2}\right)$.

Linear stability of the unperturbed system. It was observed that the radii $r$ of the orbits where the spacecraft is bounded in the attractor vary less than $10^{-5}$ during one orbital period, for all the attractors shown in Figs. 1 and 2, for the seven combinations of values for $\alpha_{0}$ and $\alpha_{1}$ used in the simulations made here. Therefore, we can assume that the orbits are circular for those cases. The frame of reference is changed to the one that rotates with the same angular velocity of the spacecraft around the Earth. Hence, in this frame of reference, which takes into account the Coriollis acceleration and other terms ${ }^{23}$, the spacecraft is in an equilibrium state placed at a fixed point. The accelerations evaluated at this point are of the order of $10^{-10} \mathrm{~m} / \mathrm{S}^{2}$ or smaller. The linear stability of this fixed point was evaluated by solving the characteristic equation of the linearized system and it is found that all of them are linearly stable, according to the definition given $\mathrm{in}^{24}$. 


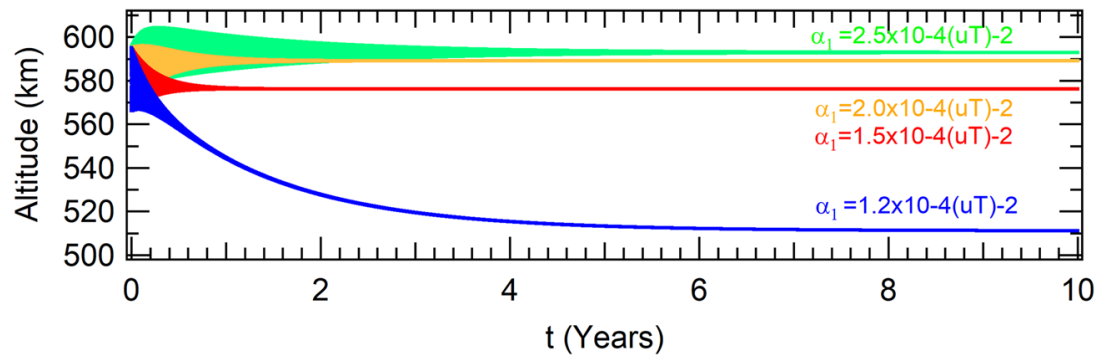

Figure 3. Perturbations due to $J_{2}$ in equatorial orbit for a thrust proportional to the radius of the orbit.

\begin{tabular}{|l|l|l|l|l|}
\hline \multirow{5}{*}{} & \multicolumn{4}{|l|}{ values for the parameter $\boldsymbol{\alpha}_{\mathbf{1}}\left(\mathbf{1 0}^{-4}(\boldsymbol{u} T)^{-2}\right)$} \\
\cline { 2 - 5 } & $\boldsymbol{\alpha}_{\mathbf{1}}=\mathbf{1 . 2}$ & $\boldsymbol{\alpha}_{\mathbf{1}}=\mathbf{1 . 5}$ & $\boldsymbol{\alpha}_{\mathbf{1}}=\mathbf{2 . 0}$ & $\boldsymbol{\alpha}_{\mathbf{1}}=\mathbf{2 . 5}$ \\
\hline$x(m)$ & 6889219.09688167 & 6954284.50657470 & 6967216.13411273 & 6971048.23011839 \\
\hline$y(m)$ & $|y|<10^{-9}$ & $|y|<10^{-9}$ & $|y|<10^{-9}$ & $|y|<10^{-9}$ \\
\hline$\dot{x}(m / s)$ & $|\dot{x}|<10^{-8}$ & $|\dot{x}|<10^{-8}$ & $|\dot{x}|<10^{-8}$ & $|\dot{x}|<10^{-8}$ \\
\hline$\dot{y}(m / s)$ & 7611.77483836 & 7575.98452101 & 7568.93140867 & 7566.84508999 \\
\hline
\end{tabular}

Table 2. Coordinates of the attractors in the phase-space of the Poincaré section using $J_{2}$ in equatorial orbits for a thrust proportional to the radius.

Brief conclusion of the unperturbed system. Those parameters $\left(\alpha_{0}, \alpha_{1}\right.$ and $\left.r_{0}\right)$ can be used to adjust the desired altitude for a specific mission. The second term of the right side of Eq. 4 is used to turn the magnitude of the thrust close to the equilibrium with the magnitude of the force due to drag, and it defines the altitude of the attractor, as can be seen in Fig. 2 by the variation of its parameter $\alpha_{0}$, while the first term of Eq. 4 defines how the spacecraft is sent to the attractor. Note, from Table 1, that the parameter $\alpha_{1}$ does not change the position of the spacecraft in the phase-space of the Poincaré section. They have almost the same coordinates in this phase-space. On the other side, note from Fig. 1 that this same parameter $\left(\alpha_{1}\right)$ highly influences the transient time, i.e., the time the spacecraft takes to reach the attractor. Besides that, it influences the oscillation during this transient, from an overdumped pattern for low values of the parameter to an underdumped one for higher values of $\alpha_{1}$. It is also possible to interpret this thrust by using two engines: a constant one that applies the major thrust correspondent to the second term of the right side of Eq. 4, and a variable one that applies the thrust correspondent to the first term of the right side of Eq. 4 .

Perturbed system. At this stage of the research, perturbations $(\vec{P})$ will be taken into consideration for a more complete study of the system. Their influence over the attractors will be investigated.

The first perturbation added to the system is given by the $J_{2}$ term of the Earth gravitational potential, which is due to its oblateness shape. The evolutions of the altitudes in time are shown in Fig. 3, for the same values of the parameters shown in Fig. 1, in the case where the orbit is in the equatorial plane of the Earth. In comparison with the unperturbed problem, the net effect of $J_{2}$ is to reduce the altitude of the orbit. This is an expected result, since the $J_{2}$ term is equivalent to one increase in the mass of the Earth, for equatorial orbits, in comparison with a spherical Earth. Although the attractor is moved to lower altitudes, the transient time to reach is approximately the same. The values of $\alpha_{1}$ between $\alpha_{1}=1.5 \times 10^{-4}\left(u T^{-2}\right)$ and $\alpha_{1}=2.0 \times 10^{-4}\left(u T^{-2}\right)$ are the ones in which the spacecraft evolves to the attractor in shorter times. The radius of the orbit changes less than $10^{-5} \mathrm{~m}$ during the period of an orbit, so the spacecraft is found in a circular orbit which coordinates in the phase-space of the Poincaré section are shown in Table 2.

In the case of polar orbits, the altitudes of the attractors have an opposite behavior in comparison with the case where the orbit is equatorial. Their altitudes are shifted to higher values. The physical explanation is easy. The $J_{2}$ term concentrates more mass around the equator of the Earth, so polar orbits fells a smaller mass acting in their dynamics. They increase as the parameter decreases, as shown in Fig. 4. Although the eccentricity of the orbit is not zero anymore, the attractor is periodic with period one, which means that the spacecraft crosses the Poincaré section with the single set of coordinates shown in Table 3.

In the case where the solar radiation pressure is taken into account, the frame of reference is changed to one that rotates with the same angular velocity of the Sun around the Earth. The perturbation effect over the altitude is shown in Fig. 5. Similarly to the perturbation due to the $J_{2}$ term for polar orbits, the attractors are moved to higher altitudes. The physical explanation is also easy to be made. The Solar Radiation Pressure acts opposite to the gravity of the Earth in the reference frame used here, when the spacecraft is in the semi-plane $x>0$. So, its effect is equivalent to a reduction of the mass of the Earth, which gives higher final altitudes for the spacecraft. It has two opposite effects in the semi-plane $x<0$, and the altitude decreases. It generates an oscilattion of the final altitude, as visible in Fig. 5. It means that a solar sail can be used to give different values for the final mean altitude and its range of oscilation of the spacecraft, acting as a passive control The attractor is also periodic with 


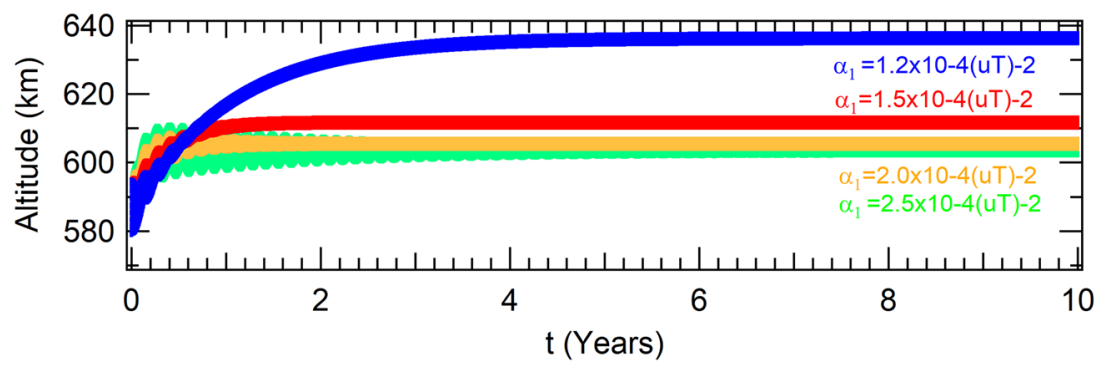

Figure 4. Effects of the perturbation due to $J_{2}$ in polar orbit for a thrust proportional to the radius of the orbit in the altitudes of the spacecraft.

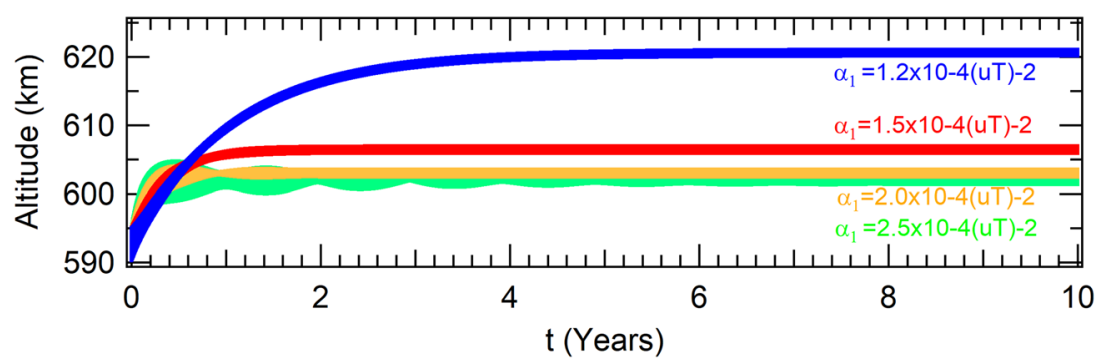

Figure 5. Effects of the perturbations due to $S R P$ for a thrust proportional to the radius in the final altitude of the orbit of the spacecraft.

\begin{tabular}{|l|l|l|l|l|}
\hline \multirow{5}{*}{} & \multicolumn{5}{|l|}{ values for the parameter $\boldsymbol{\alpha}_{\mathbf{1}}\left(\mathbf{1 0}^{-\mathbf{4}}(\boldsymbol{u} \text { T })^{-\mathbf{2}}\right)$} \\
\cline { 2 - 5 } & $\boldsymbol{\alpha}_{\mathbf{1}}=\mathbf{1 . 2}$ & $\boldsymbol{\alpha}_{\mathbf{1}}=\mathbf{1 . 5}$ & $\boldsymbol{\alpha}_{\mathbf{1}}=\mathbf{2 . 0}$ & $\boldsymbol{\alpha}_{\mathbf{1}}=\mathbf{2 . 5}$ \\
\hline$x(m)$ & 7015945.35215650 & 6991320.89415925 & 6985126.18986852 & 6983241.55544237 \\
\hline$y(m)$ & $|y|<10^{-9}$ & $|y|<10^{-9}$ & $|y|<10^{-9}$ & $|y|<10^{-9}$ \\
\hline$\dot{x}(\mathrm{~m} / \mathrm{s})$ & 0.00037892 & 0.00041423 & 0.00047159 & 0.00052294 \\
\hline$\dot{y}(\mathrm{~m} / \mathrm{s})$ & 7539.156133457 & 7552.43334311 & 7555.78451696 & 7556.80494032 \\
\hline
\end{tabular}

Table 3. Coordinates of the attractors in the phase-space of the Poincaré section when considering $J_{2}$ in polar orbits for a thrust proportional to the radius of the orbit.

\begin{tabular}{|l|l|l|l|l|}
\hline \multirow{5}{*}{} & \multicolumn{4}{|l|}{ values for the parameter $\boldsymbol{\alpha}_{\mathbf{1}}\left(\mathbf{1 0}^{-4}(\boldsymbol{u} T)^{-2}\right)$} \\
\cline { 2 - 5 } & $\boldsymbol{\alpha}_{\mathbf{1}}=\mathbf{1 . 2}$ & $\boldsymbol{\alpha}_{\mathbf{1}}=\mathbf{1 . 5}$ & $\boldsymbol{\alpha}_{\mathbf{1}}=\mathbf{2 . 0}$ & $\boldsymbol{\alpha}_{\mathbf{1}}=\mathbf{2 . 5}$ \\
\hline$x(m)$ & 6998357.61992621 & 6984120.22756220 & 6980577.78678919 & 6979473.21206146 \\
\hline$y(m)$ & $|y|<10^{-9}$ & $|y|<10^{-9}$ & $|y|<10^{-9}$ & $|y|<10^{-9}$ \\
\hline$\dot{x}(m / s)$ & -0.32090338 & -0.29640297 & -0.20906448 & -0.05888977 \\
\hline$\dot{y}(m / s)$ & 7545.73024554 & 7553.45365269 & 7555.42601747 & 7556.05885375 \\
\hline
\end{tabular}

Table 4. Coordinates of the attractors in the phase-space of the Poincaré section due to SRP for a thrust proportional to the radius.

period one in the phase-space of the Poincaré section using the rotating frame of reference, and its coordinates are shown in Table 4.

Another perturbation to be considered is the gravitational potential of the Moon. Once again, to keep the dimension of the phase-space of the Poincaré section unchanged, the evaluations were done in a frame of reference that rotates with the same angular velocity of the Moon around the Earth. In this case, the effect of the perturbation due to the gravitational influence of the Moon over the altitude of the spacecraft is shown in Fig. 6 . The coordinates of the attractors in the rotating frame of reference is shown in Table 5.

Brief conclusions of the perturbed system. The parameter $\alpha_{1}$ plays an important role in the altitude of the attractor: the higher it is, the stronger the contribution of the term $\alpha_{1}\left(r_{0}-r\right)$ of Eq. 4 to the thrust, and the closer the attractor is to $r_{0}$. The displacements of the attractors are in the direction of higher altitudes for all kinds of perturbations shown here, except in the case of equatorial orbits due to the inclusion of the $J_{2}$ term of the gravity field 


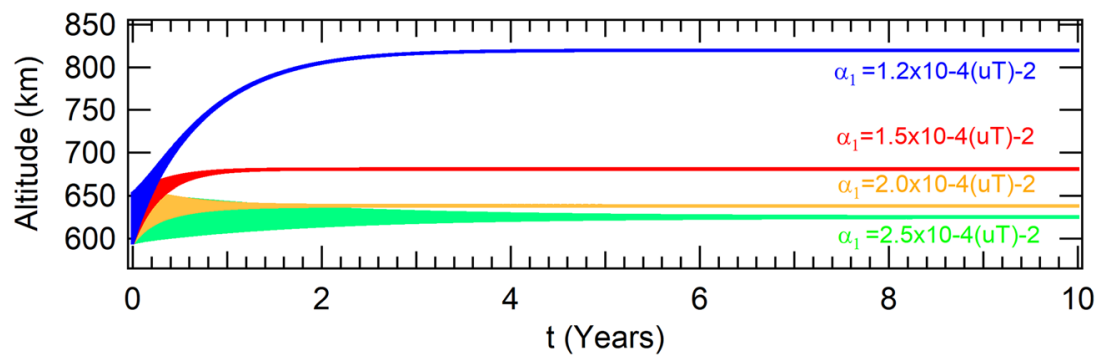

Figure 6. Effects of the perturbations due to the Moon for a thrust proportional to the radius in the final altitude of the orbit of the spacecraft.

\begin{tabular}{|l|l|l|l|l|}
\hline \multirow{5}{*}{} & \multicolumn{4}{|l|}{ values for the parameter $\boldsymbol{\alpha}_{\mathbf{1}}\left(\mathbf{1 0}^{-\mathbf{4}}(\boldsymbol{u} \mathbf{T})^{-\mathbf{2}}\right)$} \\
\cline { 2 - 5 } & $\boldsymbol{\alpha}_{\mathbf{1}}=\mathbf{1 . 2}$ & $\boldsymbol{\alpha}_{\mathbf{1}}=\mathbf{1 . 5}$ & $\boldsymbol{\alpha}_{\mathbf{1}}=\mathbf{2 . 0}$ & $\boldsymbol{\alpha}_{\mathbf{1}}=\mathbf{2 . 5}$ \\
\hline$x(m)$ & 7197877.23607033 & 7058760.78277839 & 7016200.18755208 & 7002968.32880616 \\
\hline$y(m)$ & $|y|<10^{-9}$ & $|y|<10^{-9}$ & $|y|<10^{-9}$ & $|y|<10^{-9}$ \\
\hline$\dot{x}(\mathrm{~m} / \mathrm{s})$ & -0.00117907 & -0.00093344 & -0.00052700 & -0.00004123 \\
\hline$\dot{y}(\mathrm{~m} / \mathrm{s})$ & 7422.96825977 & 7496.30004086 & 7519.167244835 & 7526.31877766 \\
\hline
\end{tabular}

Table 5. Coordinates of the attractors in the phase-space of the Poincaré section due to the Moon for a thrust proportional to the radius.

\begin{tabular}{|l|l|l|l|}
\hline \multirow{2}{*}{} & \multicolumn{3}{|l|}{ values for the parameter $\boldsymbol{\alpha}_{\mathbf{2}}\left(\mathbf{1 0}^{-\mathbf{4}}(\boldsymbol{u L} / \mathbf{u} \boldsymbol{T})\right.$} \\
\cline { 2 - 4 } & $\boldsymbol{\alpha}_{\mathbf{2}}=\mathbf{1 . 0 6 7}$ & $\boldsymbol{\alpha}_{\mathbf{2}}=\mathbf{1 . 0 6 8}$ & $\boldsymbol{\alpha}_{\mathbf{2}}=\mathbf{1 . 0 7 0}$ \\
\hline$x(m)$ & 6964560.37846288 & 6977620.96701989 & 7003778.84836227 \\
\hline$y(m)$ & $|y|<10^{-9}$ & $|y|<10^{-9}$ & $|y|<10^{-9}$ \\
\hline$\dot{x}(\mathrm{~m} / \mathrm{s})$ & $|\dot{x}|<10^{-8}$ & $|\dot{x}|<10^{-8}$ & $|\dot{x}|<10^{-8}$ \\
\hline$\dot{y}(\mathrm{~m} / \mathrm{s})$ & 7565.22821813 & 7558.14467111 & 7544.01729790 \\
\hline
\end{tabular}

Table 6. Coordinates of the attractors in the phase-space of the Poincaré section due to $J_{2}$ in equatorial orbit for thrust proportional to the radius.

of the Earth, because it increases the effective mass of the central body. The gravitational effect of the Moon has the highest influence in the net displacement from $r_{0}$, in comparison with other perturbations. The last important result is that, for all perturbed cases studied in this work, the spacecraft always evolved to periodic attractors with period one in their respective frame of reference. This can be helpful to track it, since it will always cross its respectives coordinates from time to time.

Thrust proportional to the magnitude of the velocity and inversely proportional to the radius. In this subsection, we investigate a thrust used to maintain the spacecraft in attractors at desired altitudes that is proportional to the angular velocity $v / r$. When the spacecraft if falling into the Earth, its velocity is increased and its radius is decreased, hence the magnitude of the thrust is increased and the result is that it tends to evolve to its respective attractor. In this case, the magnitude of the thrust $\tau$ is given by

$$
\tau=\alpha_{2} \frac{v}{r}
$$

The spacecraft evolves to the attractors shown in Table 6 for three different values of the parameter $\alpha_{2}$. The evolutions of the respective altitudes are shown in Fig. 7. The higher this parameter, the higher the altitude of the attractor.

Linear stability of the unperturbed system. Once again, the orbits of the spacecraft at the attractor are circular for all the three attractors shown in Fig. 7. Thus, they can be considered fixed points in appropriate rotating frames of references. By solving the characteristic equation of the respective linearized system, it was found that all these attractors are linearly unstable.

Perturbed case. In the case where the perturbation due to the $J_{2}$ term of the gravitational potential of the Earth is taken into account for an equatorial orbit, the spacecraft is found in the attractor which coordinates in the Poincaré section is given in Table 7, for the same values of the parameter $\alpha_{2}$ evaluated in the non perturbed case. The respective altitudes are shown in Fig. 8. In the case of a polar orbit, the coordinates of the attractors are shown in Table 8 and the respective altitudes are shown in Fig. 9. 


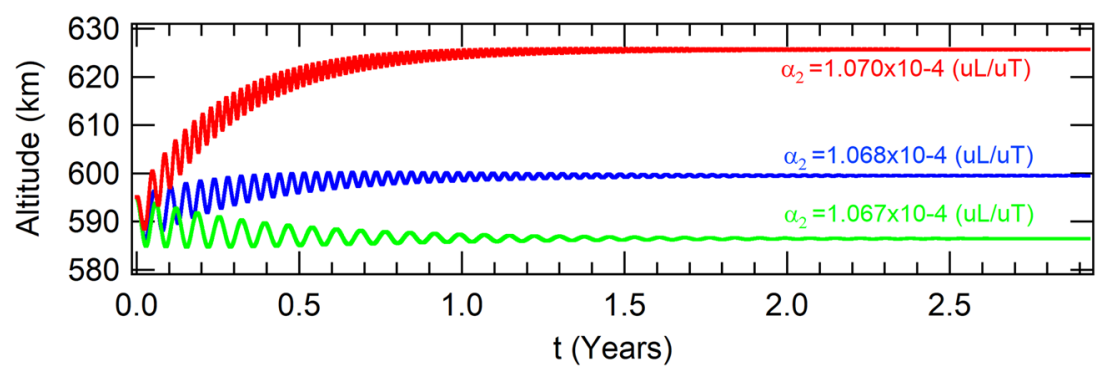

Figure 7. The altitudes of the different attractors are shown in red, blue and green, for the parameters $\alpha_{2}$ equals to $\alpha_{2}=1.070 \times 10^{-4}(u L / u T), \alpha_{2}=1.068 \times 10^{-4}(u L / u T)$ and $\alpha_{2}=1.067 \times 10^{-4}(u L / u T)$, respectively. The other parameters are $\beta=1000 \times \beta_{\text {ISS }}$ and $a_{0}=r_{0}-5 \mathrm{~km}$.

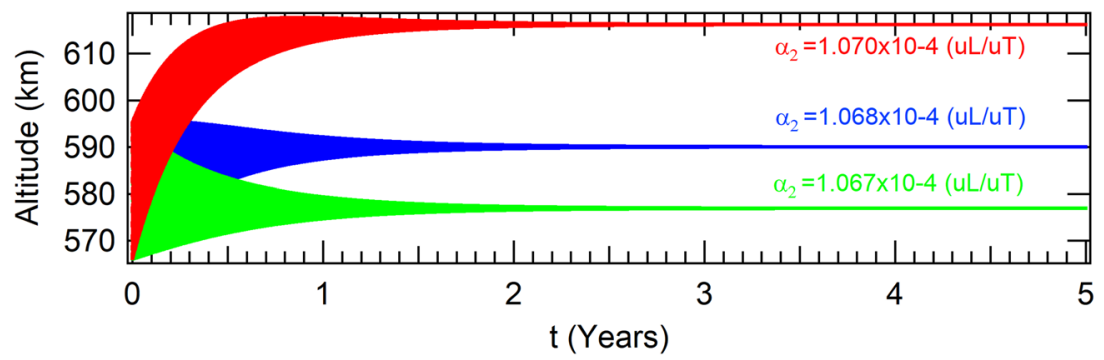

Figure 8. Perturbations due to $J_{2}$ in equatorial orbits for a thrust proportional to the velocity and inversely proportiuonal to the radius of the orbit of the spacecraft.

\begin{tabular}{|l|l|l|l|}
\hline \multirow{4}{*}{} & \multicolumn{3}{|l|}{ values for the parameter $\boldsymbol{\alpha}_{\mathbf{2}}\left(\mathbf{1 0}^{-\mathbf{4}}(\boldsymbol{u L} / \boldsymbol{u})\right)$} \\
\cline { 2 - 4 } & $\boldsymbol{\alpha}_{2}=\mathbf{1 . 0 6 7}$ & $\boldsymbol{\alpha}_{2}=1.068$ & $\boldsymbol{\alpha}_{2}=\mathbf{1 . 0 7 0}$ \\
\hline$x(m)$ & 6955061.54361013 & 6968139.95931161 & 6994333.34424211 \\
\hline$y(m)$ & $|y|<10^{-9}$ & $|y|<10^{-9}$ & $|y|<10^{-9}$ \\
\hline$\dot{x}(\mathrm{~m} / \mathrm{s})$ & $|\dot{x}|<10^{-8}$ & $|\dot{x}|<10^{-8}$ & $|\dot{x}|<10^{-8}$ \\
\hline$\dot{y}(\mathrm{~m} / \mathrm{s})$ & 7575.56022172 & 7568.42828979 & 7554.20496085 \\
\hline
\end{tabular}

Table 7. Coordinates of the attractors in the phase-space of the Poincaré section to $J_{2}$ in equatorial orbit for a thrust proportional to the velocity and inversely proportional to the radius of the orbit of the spacecraft.

\begin{tabular}{|l|l|l|l|}
\hline \multirow{2}{*}{} & \multicolumn{3}{|l|}{ values for the parameter $\boldsymbol{\alpha}_{2}\left(\mathbf{1 0}^{-4}(\boldsymbol{u L} / \mathbf{u})\right)$} \\
\cline { 2 - 4 } & $\boldsymbol{\alpha}_{2}=1.067$ & $\boldsymbol{\alpha}_{2}=1.068$ & $\boldsymbol{\alpha}_{2}=1.070$ \\
\hline$x(m)$ & 6970883.44423619 & 6983932.19757493 & 7010066.50793921 \\
\hline$y(m)$ & $|y|<10^{-9}$ & $|y|<10^{-9}$ & $|y|<10^{-9}$ \\
\hline$\dot{x}(\mathrm{~m} / \mathrm{s})$ & 0.00012198 & 0.00012164 & 0.000120963 \\
\hline$\dot{y}(\mathrm{~m} / \mathrm{s})$ & 7563.50643237 & 7556.43094902 & 7542.31956748 \\
\hline
\end{tabular}

Table 8. Coordinates of the attractors in the phase-space of the Poincaré section considering $J_{2}$ for polar orbits and a thrust proportional to the velocity and inversely proportional to the radius of the orbit of the spacecraft.

In the case where the solar radiation pressure is taken into account, the spacecraft will evolve to the attractors shown in Table 9, which altitudes evolutions in time can be seen in Fig. 10.

For the perturbation due to the gravitational attraction of the Moon, the spacecraft evolves to the attractors shown in Table 10. Its altitudes as function of time are shown in Fig. 11.

A more general model for the thrust. In this case, the thrust over the spacecraft is given by

$$
\tau=\alpha_{2} \frac{v}{r}+\alpha_{1}\left(r_{0}-r\right)+\alpha_{0} \beta v_{0}^{2}
$$




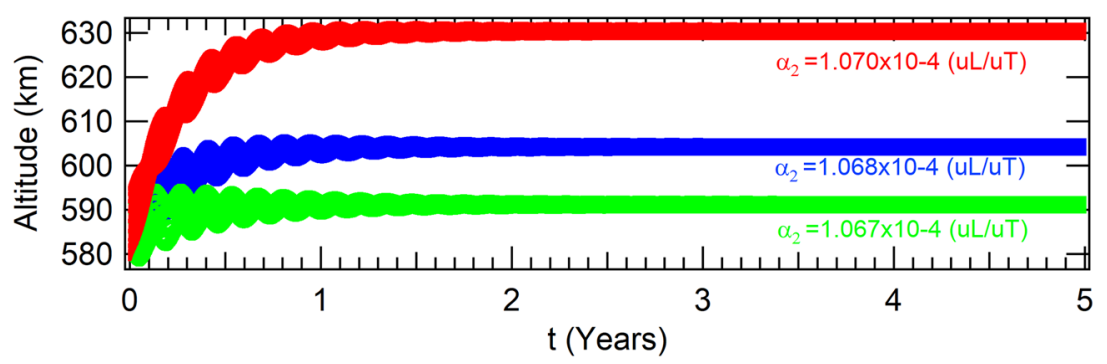

Figure 9. Perturbations due to $J_{2}$ in polar orbits for a thrust proportional to the velocity and inversely to the radius of the orbit of the spacecraft.

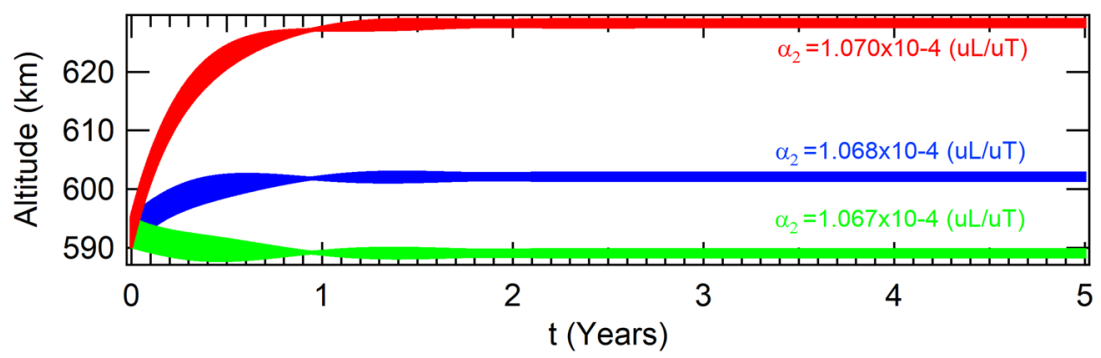

Figure 10. Perturbations due to $S R P$ for a thrust proportional to the velocity and inversely proportional to the radius of the orbit of the spacecraft.

\begin{tabular}{|l|l|l|l|}
\hline \multirow{2}{*}{} & \multicolumn{4}{|l|}{ values for the parameter $\boldsymbol{\alpha}_{\mathbf{2}}\left(\mathbf{1 0}^{-\mathbf{4}}(\boldsymbol{u L} / \mathbf{u})\right)$} \\
\cline { 2 - 4 } & $\boldsymbol{\alpha}_{2}=\mathbf{1 . 0 6 7}$ & $\boldsymbol{\alpha}_{2}=\mathbf{1 . 0 6 8}$ & $\boldsymbol{\alpha}_{\mathbf{2}}=\mathbf{1 . 0 7 0}$ \\
\hline$x(m)$ & 6966592.41369304 & 6979662.15516907 & 7005838.44220465 \\
\hline$y(m)$ & $|y|<10^{-9}$ & $|y|<10^{-9}$ & $|y|<10^{-9}$ \\
\hline$\dot{x}(m / s)$ & -0.174013958 & -0.174290219 & -0.1748698966 \\
\hline$\dot{y}(m / s)$ & 7563.02151982 & 7555.93425592 & 7541.79943999 \\
\hline
\end{tabular}

Table 9. Coordinates of the attractors in the phase-space of the Poincaré section including SRP for a thrust proportional to the velocity and inversely proportional to the radius of the orbit of the spacecraft.

\begin{tabular}{|l|l|l|l|}
\hline \multirow{2}{*}{} & \multicolumn{3}{|l|}{ values for the parameter $\boldsymbol{\alpha}_{\mathbf{2}}\left(\mathbf{1 0}^{-\mathbf{4}}(\boldsymbol{u L} / \boldsymbol{u} T)\right)$} \\
\cline { 2 - 4 } & $\boldsymbol{\alpha}_{2}=\mathbf{1 . 0 6 7}$ & $\boldsymbol{\alpha}_{2}=\mathbf{1 . 0 6 8}$ & $\boldsymbol{\alpha}_{2}=\mathbf{1 . 0 7 0}$ \\
\hline$x(m)$ & 6998127.97657272 & 7011346.92909045 & 7037823.34032799 \\
\hline$y(m)$ & $|y|<10^{-9}$ & $|y|<10^{-9}$ & $|y|<10^{-9}$ \\
\hline$\dot{x}(m / s)$ & -0.00043678 & -0.00043801 & -0.00044046 \\
\hline$\dot{y}(m / s)$ & 7528.93992007 & 7521.78798627 & 7507.52364247 \\
\hline
\end{tabular}

Table 10. Coordinates of the attractors in the phase-space of the Poincaré section including Moon for a thrust proportional to the velocity and inversely proportional to the radius of the orbit of the spacecraft.

The parameter $r_{0}$ is set to $r_{0}=r_{e}+600 \mathrm{~km}$ and the initial condition is $5 \mathrm{~km}$ below this value $\left(a_{0}=r_{0}-5 \mathrm{~km}\right)$. The other parameters are fixed in the values $\alpha_{0}=6.5 \times 10^{-2}, \alpha_{1}=1 \times 10^{-5}\left(u T^{-2}\right)$ and $\alpha_{2}=1 \times 10^{-4}(u L / u T)$ and they are modified one at a time in each color in Fig. 12. The attractors are shown in Table 11.

Linear stability of the unperturbed system. The orbits of the spacecraft at the four attractors shown in Fig. 12 can also be considered as circular ones. By solving the characteristic equation of the respective linearized system in appropriate frames of references, it was found that the attractor $A_{N}, A_{1}$ and $A_{2}$ are stable, while the attractor $A_{0}$ is unstable. 


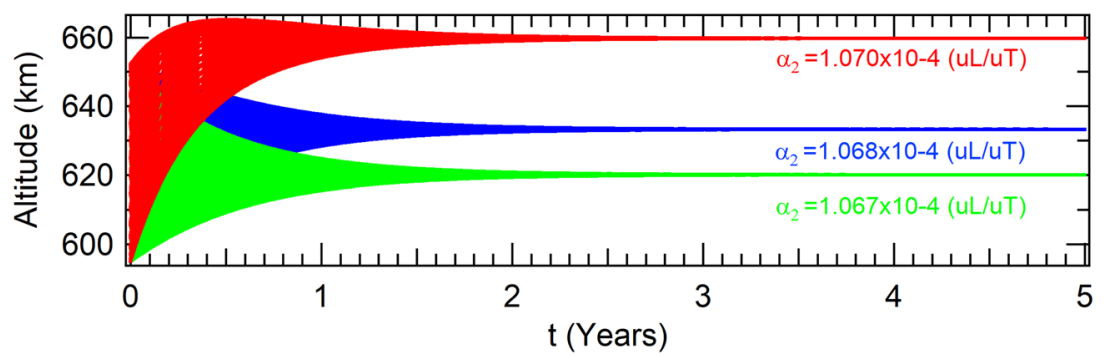

Figure 11. Perturbations due to the Moon for a thrust proportional to the velocity and inversely proportional to the radius.

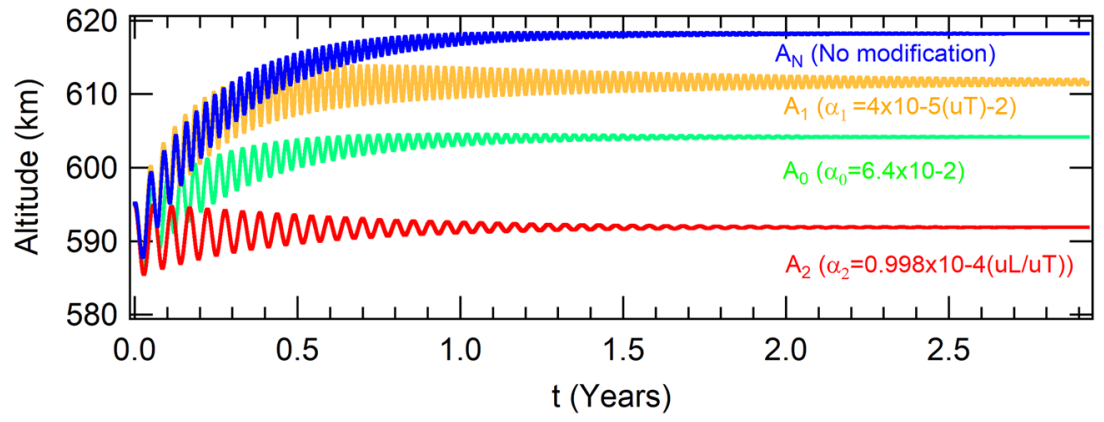

Figure 12. The evolution of the spacecraft to the attractors $A_{N}, A_{0}, A_{1}$ and $A_{2}$ as functions of time. The parameters of the attractor $A_{N}$ are $\alpha_{0}=6.5 \times 10^{-2}, \alpha_{1}=1 \times 10^{-5}\left(u T^{-2}\right)$ and $\alpha_{2}=1 \times 10^{-4}(u L / u T)$. The parameters of the attractors $A_{0}, A_{1}$ and $A_{2}$ are the same of $A_{N}$, except $\alpha_{0}, \alpha_{1}$ and $\alpha_{2}$, respectively.

\begin{tabular}{|l|l|l|l|l|}
\hline \multirow{5}{*}{} & \multicolumn{4}{|l|}{ Coordinates as functions of the parameters } \\
\cline { 2 - 5 } & no modification & $\boldsymbol{\alpha}_{\mathbf{0}}=\mathbf{6 . 4} \times \mathbf{1 0}^{-\mathbf{2}}$ & $\boldsymbol{\alpha}_{\mathbf{1}}=\mathbf{4} \times \mathbf{1 0}^{-\mathbf{5}}\left(\boldsymbol{u} \boldsymbol{T}^{-2}\right)$ & $\boldsymbol{\alpha}_{\mathbf{2}}=\mathbf{9 . 9 9 8} \times \mathbf{1 0}^{-\mathbf{5}}(\boldsymbol{u L} / \boldsymbol{u} \boldsymbol{T})$ \\
\hline$x(m)$ & 6996351.67397836 & 6982273.22670731 & 6989755.98126234 & 6970012.75529358 \\
\hline$y(m)$ & $|y|<10^{-9}$ & $|y|<10^{-9}$ & $|y|<10^{-9}$ & $|y|<10^{-9}$ \\
\hline$\dot{x}(m / s)$ & $|\dot{x}|<10^{-8}$ & $|\dot{x}|<10^{-8}$ & $|\dot{x}|<10^{-8}$ & $|\dot{x}|<10^{-8}$ \\
\hline$\dot{y}(m / s)$ & 7548.02051789 & 7555.62627132 & 7551.58092009 & 7562.26864358 \\
\hline
\end{tabular}

Table 11. Coordinates of the attractors in the phase-space of the Poincaré section.

\begin{tabular}{|l|l|l|l|l|}
\hline \multirow{5}{*}{} & \multicolumn{5}{|l|}{ Coordinates as functions of the perturbations } \\
\cline { 2 - 5 } & $\boldsymbol{J}_{2}$ Eq. & $\boldsymbol{J}_{2}$ Polar & SRP & Moon \\
\hline$x(m)$ & 6986211.37428672 & 7002984.55909160 & 6998600.80209396 & 7032733.18747531 \\
\hline$y(m)$ & $|y|<10^{-9}$ & $|y|<10^{-9}$ & $|y|<10^{-9}$ & $|y|<10^{-9}$ \\
\hline$\dot{x}(m / s)$ & $|\dot{x}|<10^{-8}$ & 0.00014044 & -0.18507844 & -0.00047047 \\
\hline$\dot{y}(m / s)$ & 7558.60669434 & 7546.13569400 & 7545.6963499 & 7510.259769 \\
\hline
\end{tabular}

Table 12. Perturbations with a more general model to thrust.

Perturbed case. Considering the values for the parameters of the "no modification" blue curve of Fig. 12, in the case where the perturbations due to the oblateness of the Earth is considered for polar and equatorial orbits, the SRP and the gravitation effect of the Moon, the spacecraft evolves to the attractors shown in Table 12. The altitudes as functions of time are shown in Fig. 13.

The higher the parameter $\alpha_{0}$, the higher the altitude of the attractor (and vice-versa), but this parameter is also related to the transient time. The lower values of $\alpha_{0}$, the higher the time to reach the attractor. The higher the parameter $\alpha_{1}$, the closer the attractor is to $r=r_{0}$, but, also, the higher its value, the higher is the amplitude of oscillations, and, as a consequence, more time is required for the system to reach the attractor. The higher the parameter $\alpha_{2}$, the higher the altitude of the attractor. It is also lower the time that the system requires to reach it. These parameters are important for a mission designer. As an example, in the case where a spacecraft needs to reach a previous determined altitude in the lower possible time, the parameter $\alpha_{1}$ should be reduced. 


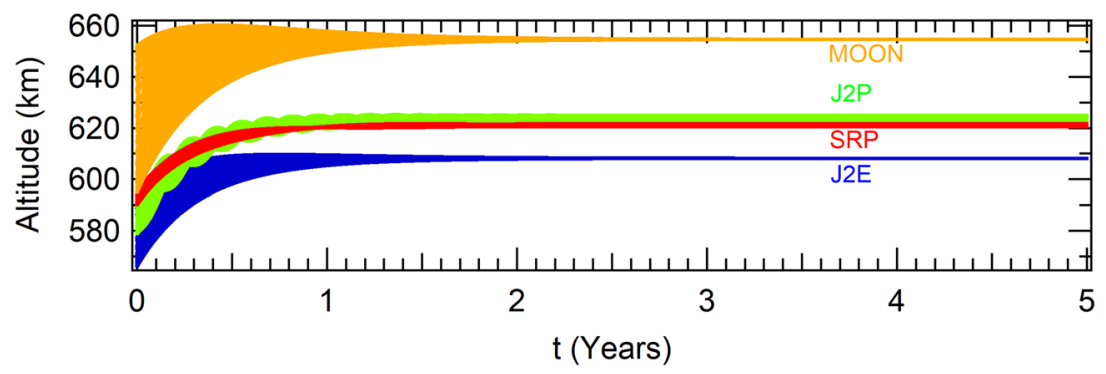

Figure 13. The influence of several types of perturbations over the attractor $A_{N}$ shown in Fig. 12, which parameters are $\alpha_{0}=6.5 \times 10^{-2}, \alpha_{1}=1 \times 10^{-5}\left(u T^{-2}\right)$ and $\alpha_{2}=1 \times 10^{-4}(u L / u T)$.

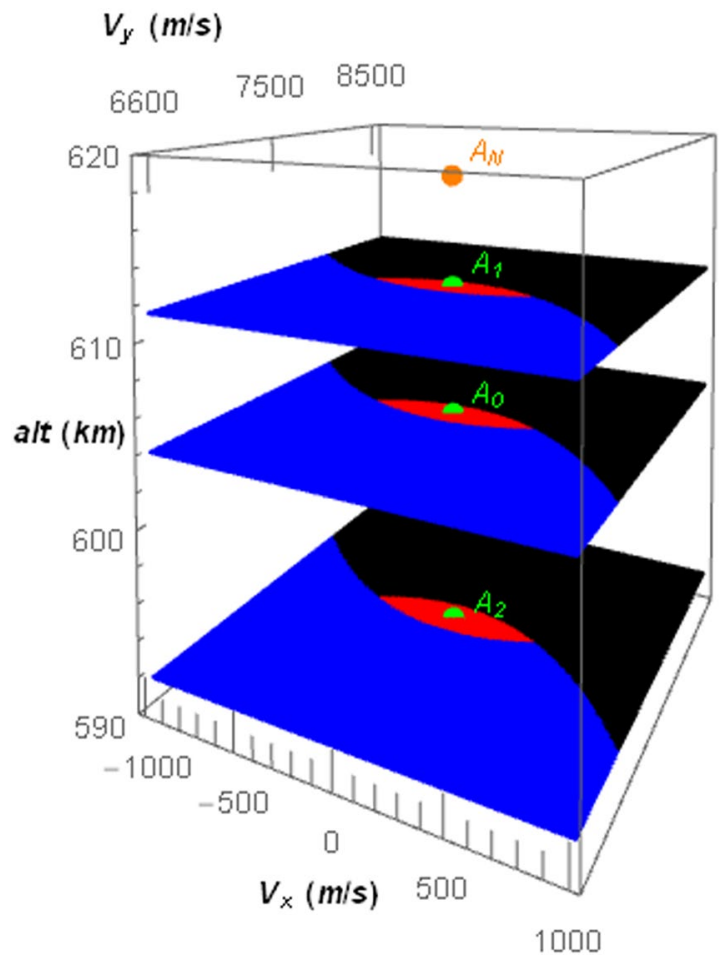

Figure 14. Planar sections of the basin of attraction (in red) of the attractor shown in orange for the parameters $\alpha_{0}=6.5 \times 10^{-2}, \alpha_{1}=1 \times 10^{-5}\left(u T^{-2}\right)$ and $\alpha_{2}=1 \times 10^{-4}(u L / u T)$ for several values of the initial altitude. The attractors for a modified parameter - $A_{0}$ for $\alpha_{0}=6.4 \times 10^{-2}, A_{1}$ for $\alpha_{1}=4 \times 10^{-5}\left(u T^{-2}\right)$ and $A_{2}$ for $\alpha_{2}=9.998 \times 10^{-5}(u L / u T)$ are shown in green. The coordinates of these attractors in the phase-space of the Poincaré section are also shown in Table 11 .

Transfers among attractors. In this section, the transfers among attractors will be studied using a basin of attraction. In the case where a spacecraft is bounded in an attractor and that it should be transferred to another altitude, the evaluation of the basin of attraction of the final attractor can show if the transfer can be done by a change of parameters.

There are several applications of this idea, like to make a transfer orbit to place the spacecraft in a different altitude to make observations from another location, rendezvouz with another spacecraft, using an altitude that requires less fuel consumption for maintenance, to make the spacecraft to reenter the atmosphere to be destroyed; or even to escape from the Earth. As an example, we suppose that a spacecraft should be transferred to an altitude $a l t=618216 \mathrm{~m}$ through the attractor described as "No modification" shown in Fig. 12 and Table 11. The parameters of the thrust must be fixed in the values $\alpha_{0}=6.5 \times 10^{-2}, \alpha_{1}=1 \times 10^{-5}\left(u T^{-2}\right)$ and $\alpha_{2}=1 \times 10^{-4}(u L / u T)$, but the transfer will succeed only if the coordinates of the spacecraft in its phase-space are within the basin of attraction of the final attractor.

In Fig. 14, several planar sections of the basin of attraction with respect to the "No modification" attractor are shown in the phase-space of the Poincaré section. The regions in red will evolve to the attractor, while the initial conditions in blue will make the spacecraft to fall in the Earth and the initial conditions in black will make the spacecraft to escape from the Earth. Numerically, the conditions in red will reach the attractor with a precision of $10^{-3}$ in position and velocities coordinates in the IS, while the conditions in blue will evolve the spacecraft to 

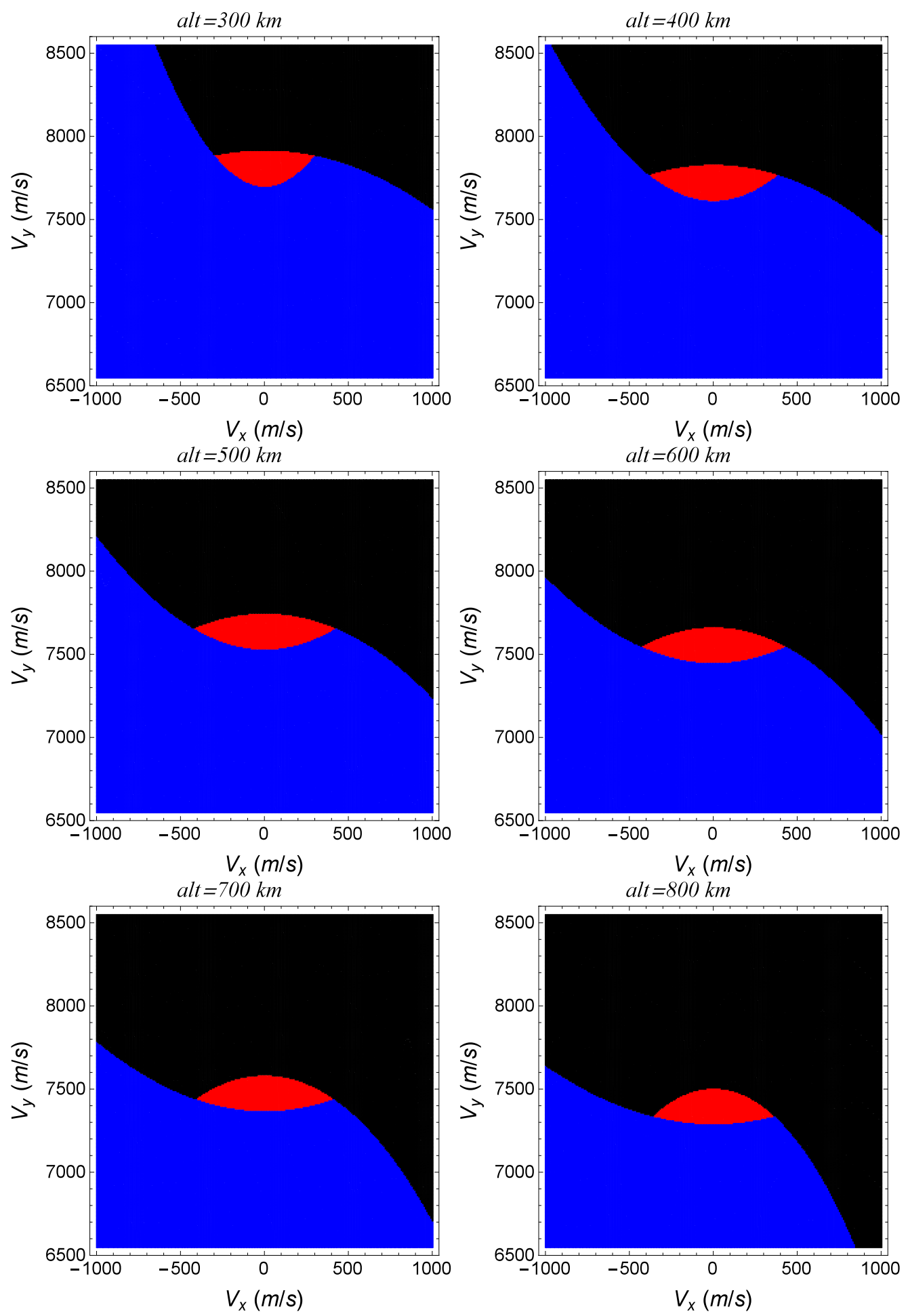

Figure 15. Basins of attraction of the attractor shown in Table 11 are shown in red for the parameters $\alpha_{0}=6.5 \times 10^{-2}, \alpha_{1}=1 \times 10^{-5}\left(u T^{-2}\right)$ and $\alpha_{2}=1 \times 10^{-4}(u L / u T)$ for several values of the initial altitude.

fall to altitudes lower than $200 \mathrm{~km}$, (that will end in reentry) in the atmosphere of the Earth, and the conditions in black reach altitudes higher than $1000 \mathrm{~km}$. Note that only the initial conditions of the spacecraft in red can evolve it to the "No modification" attractor. The coordinates in the phase-space of the attractors shown in Fig. 12 and Table 11 for several values of modified parameters are also shown in Fig. 14, which are placed in altitudes equal to 592, 604 and $612 \mathrm{~km}$. Note that these attractors are contained in the red region of the plane, which means that the spacecraft can be transferred from one of these attractors to the "No modification" attractor by a change of parameters of the thrust. In Fig. 15, planes of the basin of attraction are shown for several values of altitude. They 
represent the conditions (components of the velocity) in which a spacecraft can be set such that it will evolve to the "No modification" attractor as function of the respective altitudes.

\section{Conclusions}

In this work, it is shown how a spacecraft equipped with a thrust can be bounded in specific altitudes in LEO, taken into consideration the drag, the gravitational influence of the Earth and the Moon, the SRP, and the oblateness of the Earth for equatorial and polar orbits for several types of thrusts. In the cases where only the Earth's gravity, the drag and the thrust are taken into consideration, the orbit is circular for all the cases investigated here.

Some attractors correspond to circular orbits and they become fixed points in proper rotating frames of reference. In the case where the model to the thrust is given by Eq. (4), these fixed points are linearly stable for all the attractors shown in section 3.1 when no perturbation is taken into consideration. The attractors to where the spacecraft is evolved are unstable for the cases evaluated with the second model of the thrust shown in section 3.2 in the case where there is no perturbation over the system. The third model shown in section 3.3 contains terms that come from the first and the second model, hence, its linear stability depends on the magnitude of each term. Thus, the linear stability analyzes indicated that the first model to the thrust shown in section 3.1 is the most reliable among the three models shown in this paper.

All types of perturbations change the attractor to higher altitudes with non circular orbits, except for the case where the perturbation is given by the $J_{2}$ term in equatorial orbits. On the other side, in appropriates frames of reference, the orbit is still periodic with a single period in the phase-space of the Poincaré section, which is an important result that can be used to simplify the prediction of the motion of a spacecraft. It facilitates its tracking over the time, which can be used to predict its motion around the Earth. Note that the drag is used to help the spacecraft to evolve to attractors, therefore, their basin of attraction are also important to predict the evolution of such a spacecraft. In this sense, transfers among different attractors are also shown and investigated in the present paper, performed by a change of the parameters of the thrust. We shown that the basin of attraction of an attractor can be used to investigate the boundaries in the phase-space that the spacecraft is placed in order to transfer it from a wide range of altitudes and velocities to final altitude and velocity desired for a mission.

In astrodynamics, in the perturbation theory, each perturbation is analyzed separately to observe and to quantify the main effects of the specific perturbation along the orbit. Under the same assumption, we analyzed the influence of the drag with multiple configurations of other perturbations and initial conditions, only to see the evolution of the orbit and the time to stabilize into the attractor. The sensitivity showed to be a function of the initial altitude and the control coefficients presented, but was not too small and the results are relevant.

The continuous low thrust was applied with a constant thrust force along the time, with maximum duration of ten years. The new advances in electric propulsion allow the implementation of propulsion systems with specific impulse larger than $10.000 \mathrm{~s}$. It is expected that new advances in this technology increase the specific impulse and the thrust duration.

Of course that a limited set of parameters and perturbations is used in this work, but the method can be extended to adapt its values to specific missions, in which intrinsic perturbations must be taken into account. Additionally, only two dimensional trajectories were investigated in this work. Of course that three dimensional orbits taken into account all the perturbation together would represent a more realistic and complete system, but it is left for further researches. Note that we used specific frames of reference according to each kind of perturbations in order to turn the equations of motion explicitly independent of time. In the case where all the perturbations are taken into account, the phase-space would have one more dimension, due to this time dependence of the equations of motion plus two dimensions due to the space span in one more degree of freedom. In this case, the analyses would be much harder to interpret in the seven dimensions of the phase-space, and we also leave this task to a future work.

Received: 23 September 2019; Accepted: 6 May 2020;

Published online: 26 May 2020

\section{References}

1. Vallado, D. Fundamentals of Astrodynamics and Applications. Springer, Berlin (2007).

2. Gomes, V. M., Piñeros, J. O. M., Prado, A. F. B. A. \& Golebiewska, J. Atmospheric close approaches with the Earth considering drag and lift forces. Computational and Applied Mathematics 35(3), 817-833 (2016).

3. Piñeros, J. O. M. \& Prado, A. F. B. A. Powered aero-gravity-assist maneuvers considering lift and drag around the Earth. Astrophysics and Space Science 362(7), 120 (2017).

4. Murcia, J. O., Prado, A. F. B. A. \& Gomes, V. M. Retrograde and Direct Powered Aero-Gravity-Assist Trajectories around Mars. Revista Mexicana de Astronomia y Astrofisica 54, 143-161 (2018).

5. de Almeida, A. K. Jr., Carvalho, R. E. \& Lima, E. F. Controlling dissociation by trapping trajectories in highly energetic states. Physica A: Statistical Mechanics and its Applications 449, 101-110 (2016).

6. Liu, H. et al. Design of a cusped field thruster for drag-free flight. Acta Astronautica 126, 35-39 (2016).

7. Canuto, E., Molano-Jimenez, A., Perez-Montenegro, C. \& Massotti, L. Long-distance, drag-free, low-thrust, LEO formation control for Earth gravity monitoring. Acta Astronautica 69(7-8), 571-582 (2011).

8. Lange, B. The drag-free satellite. AIAA journal 2(9), 1590-1606 (1964).

9. Auslander, J., Bhatia, N. P. \& Seibert, P. Attractors in dynamical systems. Bol. Soc. Mat. Mex. 9, 55-66 (1964).

10. Milnor, J. On the concept of attractor. Commun. Math. Phys. 99, 177-195 (1985).

11. Strogatz, S. H. Nonlinear Dynamics and Chaos: With Applications to Physics, Biology, Chemistry and Engineering Westview Press (2000).

12. Tsien, H. S. Take-off from satellite orbit. J. of the American Rocket Society 23, 233 (1953).

13. Vallado, D. A. \& Finkleman, D. A critical assessment of satellite drag and atmospheric density modeling. Acta Astronautica $\mathbf{9 5}$, 141-165 (2014).

14. Picone, J. M., Hedin, A. E., Drob, D. P. \& Aikin, A. C. NRLMSISE-00 empirical model of the atmosphere: Statistical comparisons and scientific issues. Journal of Geophysical Research: Space Physics 12, 107 (2002). 
15. McInnes, C. R. Solar Sailing Technology, Dynamics and Mission Applications, Springer-Verlag Berlin Heidelberg, New York (2004).

16. Funase, R. et al. On-orbit verification of fuel-free attitude control system for spinning solar sail utilizing solar radiation pressure. Advances in Space Research 48(11), 1740 (2011).

17. Hu, T. et al. Switch programming of reflectivity control devices for the coupled dynamics of a solar sail. Advances in Space Research 57(5), 1147 (2016).

18. Romagnoli, D. \& Oehlschlagel, T. High performance two degrees of freedom attitude control for solar sails. Advances in Space Research 48(11), 1869 (2011).

19. Rodriguez-Solano, C. J., Hugentobler, U. \& Steigenberger, P. Adjustable box-wing model for solar radiation pressure impacting GPS satellites. Advances in Space Research 49(7), 1113 (2012).

20. DellElce, L., Arnst, M. \& Kerschen, G. Probabilistic assessment of the lifetime of low-earth-orbit spacecraft: uncertainty characterization. J. Gui Cont Dyn 38, 900-912 (2014).

21. Fehlberg, E. Classical fifth-, sixth-, seventh-, and eighth-order Runge-Kutta formulas with stepsize control NASA NTRS (1968).

22. Ogata, K. \& Yang, Y. Modern control engineering (Vol. 4). Prentice-Hall (2002).

23. Symon, K. R. Mechanics, 2ed. Rio de Janeiro: Campus Ltda (1986).

24. Boyce, W. E. \& DiPrima, R. C. Elementary Differential Equations and Boundary Value Problems 7 th ed. - John Wiley and Sons, Inc.: New York (2001).

\section{Acknowledgements}

The authors wish to express their appreciation for the support provided by the grants 406841/2016-0 and 301338/2016-7 from the National Council for Scientific and Technological Development (CNPq); grants 2016/24561-0, 2018/07377-6 and 2019/18480-5 from São Paulo Research Foundation (FAPESP), to the financial support from the National Council for the Improvement of Higher Education (CAPES) and to the National Institute for Space Research (INPE).

\section{Author contributions}

A.K. de Almeida Jr. and J.O.M. Piñeros wrote the main version of the manuscript, generate data and prepared the figures. A.K. de Almeida Jr., J.O.M. Piñeros and A.F.B.A. Prado contributed on its conception, analysis, interpretation of data and revisions.

\section{Competing interests}

The authors declare no competing interests.

\section{Additional information}

Correspondence and requests for materials should be addressed to A.K.d.A.

Reprints and permissions information is available at www.nature.com/reprints.

Publisher's note Springer Nature remains neutral with regard to jurisdictional claims in published maps and institutional affiliations.

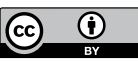

Open Access This article is licensed under a Creative Commons Attribution 4.0 International License, which permits use, sharing, adaptation, distribution and reproduction in any medium or format, as long as you give appropriate credit to the original author(s) and the source, provide a link to the Creative Commons license, and indicate if changes were made. The images or other third party material in this article are included in the article's Creative Commons license, unless indicated otherwise in a credit line to the material. If material is not included in the article's Creative Commons license and your intended use is not permitted by statutory regulation or exceeds the permitted use, you will need to obtain permission directly from the copyright holder. To view a copy of this license, visit http://creativecommons.org/licenses/by/4.0/.

(C) The Author(s) 2020 